\title{
Ecological Analysis of the COVID-19 Pandemic
}

Chrysanthus Chukwuma Sr

The Chrysanthus Centre for Future-Oriented Studies, Democracy Estate, Abakaliki, Ebonyi State, Nigeria.

Corresponding Author: Chrysanthus Chukwuma Sr, the Chrysanthus Centre for Future-Oriented Studies, Democracy Estate, Abakaliki, Ebonyi State, Nigeria.

Received date: October 31, 2020; Accepted date: January 11, 2021; Published date: January 15, 2021

Citation: C Chukwuma. (2021) Ecological Analysis of the COVID-19 Pandemic. Biomedical Research and Clinical Reviews. 3(1); DOI: $10.31579 / 2692-9406 / 032$

Copyright: (c) 2021 Chrysanthus Chukwuma, This is an open-access article distributed under the terms of the Creative Commons Attribution License, which permits unrestricted use, distribution, and reproduction in any medium, provided the original author and source are credited.

\section{Abstract}

Economic, sociological, natural, anthropogenic constructed systems and associated ambients have been enmeshed in the untoward impacts of the COVID-19 pandemic. Environment, health and quality of life should remain of immediate concern in governmental policy and governance. In this COVID-19 era, the diminished introduction of pollutants and the decreased exacerbation of contaminants in the ecosystem due to drastic reduction in economic and industrial production have perspicuously posed less threat and aberration to biodiversity, ecological and environmental systems. The conceptual ecological analysis of the COVID-19 trajectories exposes the dire consequences of the unpreparedness of nature and humans in this pandemic era. It is important to elucidate the functionalities of anthropogenic activities in environmental degradation and the inextricable-linkage between pandemics, ecosystem deterioration and health as pertinent to animal, human, plant, land usage, water and biodiversity. The conspiracy theories, ecological fallacy and mass hysteria of COVID-19 emergence stem from perspicuous extensive shirking of responsibilty regarding domestic and global environmental health by the public and private sectors of production, consumption, administration and governance.

Keywords: comorbidities; environmental health; ecological fallacy; biodiversity; anthropogenic activities; socioeconomic disruptions; mass hysteria

\section{Introduction}

The COVID-19 outbreak has assumed a global catastrophic stance, with the World Health Organization (WHO) designating it a World health emergency on 30 January, 2020. The easy and peculiar dissemination and accelerated transmissibility of the etiological agent culminated in the production of inordinate quantities of medical and environmental solid, liquid and gaseous/atmospheric wastes spewed into the biosphere. A vast majority of the world's population have been restricted in their motion/mobility in order to harness or curb the dissemination and transmission of COVID-19 and reduce contracting the disease as not to face untoward morbidity and sequelae with imminent mortality. Anthropogenic activities are being restricted during these COVID-19 episodes following the lockdown of social and economic way of life. The depicted disruptions of normal human behaviours with concomitant increased morbidity and mortality, staggered introduction of quarantine and isolation centres as well as self-isolation, job dissipation, increased unemployment hazard and elevated uncertainty and healthcare costs and supplies have thrown the world into an unprecedented hardship. Due to the uncertainty of drug treatments against the novel coronavirus, there have been expansive anecdotal information and communications needs in the administration of appropriate therapeutic regimen for symptoms in COVID-19 patients [1]

Also, COVID-19 has restricted the latitude, economic and ecological potentialities of researchers to venture into the field for data and sample collation as well as instrumentation check to explicate the sudden emergence and accelerated dissemination of the virus in certain parts of the world in perspicuous differentials or contrasts to other regions $[1,2]$.

\section{Environmental health perspectives}

Diverse environmental attributes govern the outbreak and dissemination of epidemic or pandemic occurrences with concomitant environmental feedbacks. The COVID-19 or novel coronavirus disease with its rapid and expansive spatiotemporal distribution and complex sequelae presented as a global pandemonium undergirded with ecological fallacy and mass hysteria [3]. Countries responded with diverse measures, such as the application of face masks, hand washing, social distancing and other environmental, health and healthcare measures which assisted to ameliorate the disaster in certain instances. Otherwise, the COVID-19 pandemic has been accompanied by dissipation of the ingress of income, adverse impacts of the disease and comorbidities. Anthropogenic activities were mainly determinants and risk factors in the harnessing and curbing of COVID-19 globally [4]. Anthropogenic activities and natural occurrences as well as multidimensional phenomena have been inextricably related in the resolution of the conflicting presence of the COVID-19 pandemic [5].

Numerous environmental impacts of the COVID-19 pandemic include environmental perturbation and deterioration from anthropogenic activities and uncontrolled natural interventions. These have ostensibly retarded social, economic and industrial processes, consumption, motion, natural resources, as well as decreased contaminant and pollutant emissions. However, atmospheric carbon dioxide and nitrogen oxide 
emissions have incessantly become elevated. Processes for ecosystem and biodiversity conservation almost reached diminutive capacity. These have culminated in inter alia increased unwholesome and sporadic solid wastedumping with resultant unsustainable way of existence. Also, there is associated abrogation of crucial features on global environmental governance regarding the climate change and biodiversity "super year" [6].

\section{Climate and ecological fallacy?}

Despite certain measures, such as face mask-accoutrements, handwashing procedures and social distancing, there is extant grave concerns of an emergent coronavirus mutation/new strain having elevated virus load that ostensibly overwhelms extant protective devices, and are extensively contagious but not more deadly or intensively hazardous than the extant COVID-19 [7]. With the concomitant lifestyle alterations emanating from socioeconomic retardation and dissipation of economic livelihood, sudden diminution and stagnation of leisure and recreational activities, these disruptions have culminated in decreased air/atmospheric, water and land aberration [8]. Following the gradual decline in atmospheric pollution, it is evident that there are decreased reports of the debilitative effect of COVID-19 infections of direct atmospheric pollution-to-human in lieu of person-to-person in select geographical regions [9]. The novel coronavirus that generated clusters of grave pneumonia mortality presenting clinical characteristics mimicking SARS-COV [10] has ostensibly declined in comparison to the incipient stages. Also, the ecological fallacy that associates weather status, climatic conditions, geographical areas, temperature, humidity and atmospheric effects in the preponderance and exacerbation of COVID-19 seem not to provide evidence that warmer climates are not susceptible to the rapid dissemination and transmission of COVID-19 [11]. An ecological study [12] suggested that an exact correlation between ambient temperature and COVID-19 transmissibility be elucidated within systematic and comprehensive conditions for non-linear association as evidenced in influenza using an extended observation and veritable evidence for further tests and verification. The impact assessment of disparate socioecological risk factors and determinants on the spatial distribution of the COVID-19 pandemic depicted that climatic attributes do not significantly correlate to COVID-19 risk.

\section{Urbanization and socioeconomic factors}

However, socioeconomic factors, vulnerable populations and population density correlated with COVID-19 risk [13]. Also, urban conurbation and deficient physical activity predisposed to COVID-19 morbidity and mortality [14]. The necessary response involves diverse viewpoints to elucidate the evolving natural history of SARS-CoV-2 infection due to its rapid and easy transmissibility in urban cornubations [15]. New York City presented as the preponderant USA urban cluster of the COVID-19 pandemic with varied localities subjected to disparate nuclei of incidents. Potential socioeconomic attributes tended to pertain to the neighbourhood spatiotemporal variations in the positivity rate of the COVID-19 test [16].

\section{Environmental health and biodiversity}

Biodiversity undergirds present and future economic abundance, human health, well-being and welfare [17]. It is pertinent that nations inculcate and adhere to biodiversity in all COVID-19 concerns, sustainable and restorative processes. Biodiversity protection is crucial in confronting any imminent pandemic. Certain countries or regions have integrated biodiversity paradigm in disparate COVID-19 policy and governance which invariably includes ecosystem restoration, strengthening environmental regulations, enhancing stimulus measures and biodiversity conservation [18]. The variation between socio-economic and environmental parameters depicts the complexity of interactions associated with global supply chains, spillover impacts encompassing manufacturing, tourism and transportation in the industry sectors. These need to be examined to rapidly stimulate global economy for society and environment in the present dispensation and in the future of post-COVID19 [19]. Numerous anthropogenic activities are detrimental to biodiversity, landscape and environmental heterogeneity, with expansive propensity for the dissemination of emerging and re-emerging diseases $[20,21]$. The positive environmental health effects due to stringent environmental and health activities serve as instances in way of life in which necessary behavioral changes pose positive impacts towards biodiversity and ecosystems for the present and future [22].

\section{Interactions with infectious and noninfectious diseases}

COVID-19 and non-communicable disease interactions are likely to elevate the global encumbrance of both disorders, severally or jointly [2325 . The COVID-19 pandemic has increased communicable disease research model of which certain of these studies inculcate environmental parameters. These include issues regarding spatiotemporal transmissions, comorbidities with environmentally-inextricably linked respiratory disorders, and the potential for predictive attributes in information and communication needs [1] for future local and global policies to treat, harness and curb viral transmission. Platforms exist to access environmental data which are conventionally applicable in communicable or infectious diseases with comprehensive climate monitoring data [26, 27] and ecological analysis of the COVID-19 pandemic and other such presentations.

\section{Discussion}

The COVID-19 pandemic invariably provide the latitude for a local and global re-think on the restoration and sustainability of socioeconomic activities, ecosytem and species dissipation, land and environmental degradation, investment in nature for the present and future [28]. Researchers suggest the emphasis and pertinence of prompt, stringent decision-making processes and structures to harness and curb the COVID-19 pandemic [29]. Within this framework is associated governance and administration of unintended consequences and potent policies embracing sustainable transitions, with the main objective being how to predict the outbreak size, inflection points, clusters and epicentres [30]. Another aim is evaluation of the effectivity or efficacy of intervention evolution and self-protection devices, risk estimation of the inchoate disruption of control or regulatory measures or procedures and predicting the spatiotemporal trends based on reviewed and published data and nascent mathematical modeling [20,31]. The etiology of the emergence and dissemination of COVID-19 and other zoonoses must be elucidated to prevent and obliterate fulminating infectious diseases as well as intelligently respond to future disease eruptions by inhibiting or suppressing the transmission link and eradicating the novel coronavirus and its imminent mutagens.

With the easement of the COVID-19 pandemic or restrictive measures, excoriating pressure on the ecosystem will rapidly resume with minimally constrained risks to health and biodiversity as recovery investments are allocated to polluting commercial activities with diminished pecuniary and hunger [32] stimuli for green investment. Public and private institutions must restore the economy to re-create and fill employment lacunae, food production and accessibility [32] to obviate hunger, sustain the atmospheric quality induced by COVID-19, and enhance better land, water and sanitation. In addition, institutions must improve waste management and biodiversity restoration and protection, provide for diminutive greenhouse gas emissions with concomitant decreased vulnerability and susceptibility with elevated resilience and sustainability for the future. 


\section{Conclusion}

There are extant immense impacts of the COVID-19 pandemic across countries or regions within disparate contexts. The paucity of informantion and communication have resulted in the acquisition of merely anecdotal data in most instances. There is expansive latitude for further investigation in all facets, and especially the potential effects of COVID-19 on restoration and sustainability with particular reference to the environment, health and socio-economic development as related to biodiversity, landuse, water, air, quality and way of life as well as policy making, administration and governance. The obliteration or suppression of COVID-19 and its concomitant sequelae are achievable via the knowledge of constituting an integral component where the COVID-19 pandemic, other emerging and reemerging infectious diseases will not continue to be of ecological impertinence.

\section{References}

1. Chukwuma Sr C. (2020) Information and communication needs in the administration of ibuprofen in the treatment of symptoms in COVID-19 patients. J. Med. Res. 6(4): 128-130.

2. Xiao-Guang Y, Xue-Feng S, Li RYM, Crabbe MJC, Mi L et al. (2020) Risk prediction and assessment duration, infections and death toll of the COVID-19 and its impact on China's economy. J Risk Fin Manage; 13(4): 66.

3. Dubey S, Biswas P, Ghosh R, Carl Lavie et al. (2020) Psychosocial impact of COVID-19. Diabetes \& Metab Synd Clin Res \& Rev; 14(5): 779-788.

4. Cheval S, Adamescu CM, Georgiadis T, Herrnegger M, Piticar A, Legates DR. (2020) Observed and potential impacts of the COVID-19 pandemic on the environment. Int J Environ res Public Health. 17(11): 4140.

5. Sun J, He W-T, Wong I, Lai A, Ji X, Zhau X, et al. (2020) Epidemiology, evolution, and cross-disciplinary perspectives. Trends Mol. Med.

6. Geneva Environment Network. Update: COVID-19 and the Environment. October 2020.

7. Mooney C, Achenbach J, Fox J. (2020) Massive genetic study shows coronavirus mutating and potentially evolving amid rapid U.S. Spread. The Washington Post - Health September 23 $\operatorname{arc} 404$.

8. Saeida S, Rawtani D, Hussain CM. (2020) Environmental perspective of COVID-19. Sci Total Environ. 728: 138870.

9. Barcelo D. (2020) An environmental and health perspective for COVID-19 outbreak: Meteorology and air quality influence, sewage epidemiology indicator, hospital discongestion, drug therapies and recommendations. J Environ Chem Eng. 8(4): 104006

10. Chaolin H, Yeming W, Xingwang L, Lili R, Jianping Z, Yi Y, et al. (2020) Clinical features of patients infected with 2019 novel coronavirus in Wuhan, China. Lancet. 395: 497-506.

11. Zhou Y, Jingui X. (2020) Association between ambient temperature variation and humidity on the death of COVID-19 in Wuhan, China. Sci Total Environ. 724.

12. Ran J, Zhao S, Han L, Liao G, Wang K, Wang MH, He D. (2020) A re-analysis in exploring the association between temperature and COVID-19 transmissibility: An ecological study with 154 Chinese cities. Eur Res J.

13. Su D, Chen Y, He K, Zhang T, Tan M, Zhang Y, Zhang X. (2020) Influence of socioecological factors on COVID-19 risk: a cross-sectional study based on 178 countries/regions worldwide.

14. Urashima M, Otani K, Hasegawa Y, Akutsu T. (2020) BCG vaccination and mortality of COVID-19 across 173 countries: An ecological study. Int J Env Res Public Health. 17(15): 5589.

15. De Ver Dye T, Muir E, Farovitch L, Siddiqi S, Sharma S. (2020) Critical medical ecology and SARS-CoV-2 in the urban environment: a pragmatic dynamic approach to explaining and planning for research and practice. Infectious Dis Poverty.

16. Whittle RS, Artiles AD. (2020) An ecological study of socioeconomic predictors in detection of COVID-19 cases across neighbourhoods in New York City. BMC Med 18, 271.

17. Chukwuma Sr C. (1996) Perspectives for a sustainable society. Env Manage Health. 7(5): 5-20.

18. OECD. (2020) OECD Policy Responses to Coronavirus (COVID-19). Biodiversity and the economic response to COVID-19. Ensuring a green and resilient recovery.

19. ScienceDaily. (2020) Socio-economic environmental impacts of COVID-19 quantified. University of Sydney.

20. Chukwuma Sr C. (2018) Bioinformatics-base and determinants of the spatiotemporal variations of emerging and re-emerging infectious diseases. Journal of Ancient Diseases and Preventive Remedies. 06(02).

21. CIRAD. COVID-19 The environmental origins of the pandemic.

22. El Zowalaty ME, Young SG, Jarhult JD. (2020) Environmental impact of the COVID-19 pandemic - a lesson for the future. Infection Ecology \& Epidemiology.

23. Chukwuma Sr C. (2020) The complexxity of communicable and non-communicable diseases in vulnerable populations. Adv Bioeng Biomed Sci Res.

24. Chukwuma Sr C. (2020) Aging, cellular senescence and diabetes mellitus: Clinicopathological correlates, trends and targets. CPQ Medicine.

25. Chaturvedi S, Gorelick P. (2020) The interplay between COVID-19 and non-communicable diseases. J Stroke \& Cardiovasc Dis; 29(9): 105104.

26. NOAA Climate. What environmental data are relevant to the study of infectious disease like COVID-19.

27. Al-Rousan N, Al-Najjar H. (2020) The correlation between the spread of COVID-19 infection and weather variables in 30 Chinese Provinces and the impact of Chinese Government Mitigation Plans. European Review for Medical and Pharmacological Sciences. 24(8): 4565-4571.

28. McElwee P, Turnout E, Chiroleu-Assouline M, Clapp J, Isenhour C, Jackson T et al. (2020) Ensuring a post-COVID-19 economic agenda tackles global diversity loss. One Earth.

29. Siquerva CAdS, Freitas YNLd, Cancela MdC, Carvalho M, Oliveras-Fabregas A, de Souza DLB. The effect of lockdown on the outcomes of COVID-19 in Spain: An ecological study. PLoS ONE 15(7).

30. Duan Q, Wu J, Wu G, Wang Y-G. (2020) Predication of inflection point and outbreak size of COVID-19 in new epicentres.

31. Wan H, Cui JA, Yang G-J. (2020) Risk estimation and prediction of the transmission of coronavirus disease-2019 (COVID-19) in the mainland of China excluding Hubei province. Infect Dis Poverty. 